\title{
Influence of electron-electron drag on piezoresistance of $\boldsymbol{n}$-Si
}

\author{
I.I. Boiko \\ V. Lashkaryov Institute of Semiconductor Physics, NAS of Ukraine, \\ 45, prospect Nauky, 03028 Kyiv, Ukraine \\ E-mail: igorboiko@yandex.ru; phone: (044)236-5422
}

\begin{abstract}
Piezoresistance of $n$-Si is considered with due regard for inter-valley drag. It has been shown that inter-valley drag gains the piezocoefficient and diminishes the mobility. In the region of nondegenerate carriers, the effect of drag increases when the carrier concentration rises and temperature falls.
\end{abstract}

Keywords: silicon, balance equation, mobility, piezoresistance, inter-valley drag.

Manuscript received 14.05.10; accepted for publication 16.03.11; published online 30.06.11.

\section{Introduction}

In crystals with one and only simple band, the electronelectron scattering does not change the total momentum of carriers and therefore does not give a direct, independent contribution to the conductivity. Quite another situation we have for crystals with a composite band structure. There the conductivity of crystal can be essentially influenced by mutual drag of carriers that belong to different partial bands or valleys (see Refs. $[1,2])$. In particular, the inter-valley drag can sufficiently diminish the electron mobility of $n$-Si and $n$ Ge at low temperatures. The reason of that is a principal difference between scattering of band electrons from some valley on fixed charged impurities or equilibrium phonons and scattering on nonequilibrium electrons from another valley, where divergence of equilibrium differs. Now, we have some right to hope that the intervalley drag in multy-valley semiconductors can noticeably influence not only conductivity but piezoresistance as well. In this article, we again will pay the main attention to the region of low temperatures where Coulomb scattering is not damped by collisions of electrons with phonons. We restrict here our calculations by charged impurities and acoustic phonons as an external scattering system.

\section{Balance equations}

In Refs [1, 2], the set of balance equations obtained as a first momentum of quantum kinetic equations was presented:

$e \vec{E}+\vec{F}^{(a)}+\sum_{b=1}^{6} \vec{F}^{(a, b)}=0 \quad(a=1,2, \ldots, 6)$.
Here, the symbol $a$ numerates different valleys; $\vec{E}$ is the applied electric field; the resistant force

$$
\begin{aligned}
& \vec{F}^{(a)}=-\frac{e^{2}}{(2 \pi)^{6} n_{a}} \int d^{3} \vec{k} \int \vec{q} d^{3} \vec{q} \times \\
& \times \int d \omega \delta\left(\hbar \omega-\varepsilon_{\vec{k}}^{(a)}+\varepsilon_{\vec{k}-\vec{q}}^{(a)}\right)\left\{f_{\vec{k}}^{(a)}-f_{\vec{k}-\vec{q}}^{(a)}+\left[f_{\vec{k}}^{(a)}\left(1-f_{\vec{k}-\vec{q}}^{(a)}\right)+\right.\right. \\
& \left.\left.+f_{\vec{k}-\vec{q}}^{(a)}\left(1-f_{\vec{k}}^{(a)}\right)\right] \tanh \left(\hbar \omega / 2 k_{B} T\right)\right\}\left[\left\langle\varphi_{(I)}^{2}\right\rangle_{\omega, \vec{q}}+\left\langle\varphi_{(p h)}^{2}\right\rangle_{\omega, \vec{q}}\right]
\end{aligned}
$$

is related to interaction of band carriers with charged impurities located uniformly in space; $\left\langle\varphi_{(I)}^{2}\right\rangle_{\omega, \vec{q}}$ and $\left\langle\varphi_{(p h)}^{2}\right\rangle_{\omega, \vec{q}}$ are Fourier components of the correlator of impurity and phonon scattering potentials.

The drag force

$\vec{F}^{(a, b)}=\frac{e^{4} \hbar}{4 \pi^{6} n^{(a)}} \int \vec{k} d^{3} \vec{k} \int d^{3} \vec{k}^{\prime} \times$

$\times \int d^{3} \vec{q} \frac{1}{q^{4}} \frac{\delta\left(\varepsilon_{\vec{k}}^{(a)}-\varepsilon_{\vec{k}-\vec{q}}^{(a)}-\varepsilon_{\vec{k}^{\prime}}^{(b)}+\varepsilon_{\vec{k}^{\prime}-\vec{q}^{\prime}}^{(b)}\right)}{|\varepsilon(\omega=0, \vec{q})|^{2}} \mathrm{Y}_{a b}\left(\vec{k}, \vec{k}^{\prime}, \vec{q}\right)$,

$Y_{a b}\left(\vec{k}, \vec{k}^{\prime}, \vec{q}\right)=f_{\vec{k}-\vec{q}}^{(a)}\left(1-f_{\vec{k}}^{(a)}\right) f_{\vec{k}^{\prime}}^{(b)}\left(1-f_{\vec{k}^{\prime}-\vec{q}}^{(b)}\right)-$

$-f_{\vec{k}}^{(a)}\left(1-f_{\vec{k}-\vec{q}}^{(a)}\right) f_{\vec{k}^{\prime}-\vec{q}}^{(b)}\left(1-f_{\vec{k}^{\prime}}^{(b)}\right)$

relates to interaction between drifting carriers from $a$ and $b$-valleys.

Here, $n^{(a)}$ and $\varepsilon_{\vec{k}}^{(a)}$ are the concentration and dispersion law for electrons from $a$-valley. For undeformed crystal of $n$-Si, we have (see Fig. 1): 
$\varepsilon_{\vec{k}}^{(a)}=\frac{\hbar^{2}}{2}\left(\frac{k_{x}^{2}}{m_{x x}^{(a)}}+\frac{k_{y}^{2}}{m_{y y}^{(a)}}+\frac{k_{z}^{2}}{m_{z z}^{(a)}}\right)$.

For two valleys $(a=1$ and 4$)$ $m_{z z}=m_{\|}, m_{x x}=m_{y y}=m_{\perp}$; for two valleys ( $a=2$ and 5) $m_{x x}=m_{\|}, m_{z z}=m_{y y}=m_{\perp}$ and for two valleys $\left(a=3\right.$ and 6) $m_{y y}=m_{\|}, \quad m_{x x}=m_{z z}=m_{\perp}$.

Therefore,

$$
\begin{aligned}
& \varepsilon_{\vec{k}}^{(1,4)}=\frac{\hbar^{2}}{2}\left(\frac{k_{x}^{2}+k_{y}^{2}}{m_{\perp}}+\frac{k_{z}^{2}}{m_{\|}}\right) ; \varepsilon_{\vec{k}}^{(2,5)}= \\
& =\frac{\hbar^{2}}{2}\left(\frac{k_{z}^{2}+k_{y}^{2}}{m_{\perp}}+\frac{k_{x}^{2}}{m_{\|}}\right) ; \varepsilon_{\vec{k}}^{(3,6)}=\frac{\hbar^{2}}{2}\left(\frac{k_{x}^{2}+k_{z}^{2}}{m_{\perp}}+\frac{k_{y}^{2}}{m_{\|}}\right) .
\end{aligned}
$$

The screening dielectric function for quasi-elastic collisions possesses the form $\varepsilon(\omega, \vec{q})=\varepsilon_{L}+\Delta \varepsilon_{e}(\omega=0, \vec{q})$, where $\varepsilon_{L}$ is the dielectric constant of crystal lattice and $\Delta \varepsilon_{e}(\omega, \vec{q})$ is the contribution of band electrons to the total dielectric function. For convenience, we will use the following form:

$$
\Delta \varepsilon_{e}(0, \vec{q})=\varepsilon_{L} q_{0}^{2}(\vec{q}) / q^{2} .
$$

Then,

$$
\left\langle\varphi_{(I)}^{2}\right\rangle_{\vec{q}, \omega}=\frac{32 \pi^{3} e^{2} n_{I}}{\varepsilon_{L}^{2}\left[q^{2}+q_{0}^{2}(\vec{q})\right]^{2}} \delta(\omega),
$$

$\left\langle\delta \varphi_{(p h)}^{2}\right\rangle_{\vec{q}, \omega}=\Xi_{A}^{2} \frac{2 \pi k_{B} T}{e^{2} \rho s^{2}} \delta(\omega)$.

Here, $n_{I}$ is the concentration of charged impurities; $\Xi_{A}=\Xi_{d}+(1 / 2) \Xi_{u}$, where $\Xi_{d}$ and $\Xi_{u}$ are dilation and shear deformation potential constants (see, for example, Refs. [6] and [7]). The form (7) corresponds to the approximation of quasi-elastic collisions.

The screening plays a significant, even appointing role in the area of small transferred vectors $q$; therefore, instead of $q_{0}^{2}(\vec{q})$, we can use the following approximate expression (see Ref. [1]):

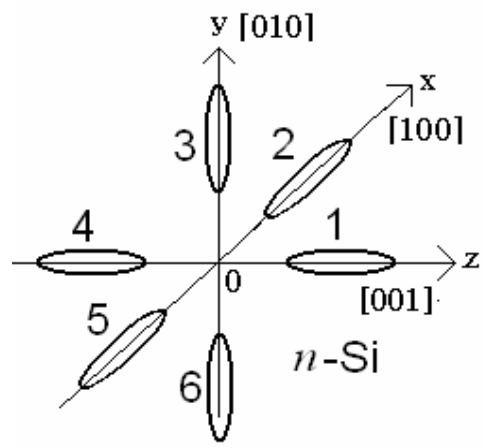

Fig. 1. Band structure of $n$-silicon. $q_{0}^{2}(q) \rightarrow q_{0}^{2}(0)=\frac{12 e^{2} m_{\|}^{3 / 2} \sqrt{2 k_{B} T}}{\hbar^{3} L \sqrt{\pi} \varepsilon_{L}} F_{-1 / 2}(\eta)$.

Here, the Fermi-integral

$F_{r}(\eta)=\frac{1}{\Gamma(r+1)} \int_{0}^{\infty} \frac{w^{r} d w}{1+\exp (w-\eta)}$,

$L=m_{\|} / m_{\perp}, \Gamma$ is the gamma-function, $\eta=\varepsilon_{F} / k_{B} T$ is the dimensionless Fermi-energy. The form (7) is valid for deformed crystal, if one uses linear approximation over deformation. For nondegenerate carriers

$q_{0}^{2}(0)=\frac{4 \pi e^{2} n}{\varepsilon_{L} k_{B} T}$.

To calculate drift velocities $\vec{u}^{(a)}$ of electrons from $a$ group, we accept the model of non-equilibrium distribution functions as Fermi functions with the argument containing shift of velocity $\vec{v}^{(a)}(\vec{k})=\hbar^{-1}\left(\partial \varepsilon_{\vec{k}}^{(a)} / \partial \vec{k}\right)$ by the correspondent velocity $\vec{u}^{(a)}$ :

$f_{\vec{k}}^{(a)}=f^{0(a)}\left(\vec{v}^{(a)}(\vec{k})-\vec{u}^{(a)}\right) \quad(a=1,2, \ldots, 6)$.

Here, $f^{0(a)}\left(\vec{v}^{(a)}(\vec{k})\right)=f_{0}{ }^{(a)}(\varepsilon)$ is the equilibrium distribution function for $a$-carriers. Drift velocities $\vec{u}^{(a)}$ are proportional to partial densities of currents $\vec{j}^{(a)}$ :

$\vec{u}^{(a)}=\frac{1}{e n^{(a)}} \vec{j}^{(a)}$.

Here, $n^{(a)}$ is the concentration of electrons in the $a$ valley. The density of total current:

$\vec{j}=\sum_{a=1}^{6} \vec{j}^{(a)}$.

Using the forms (10) and carrying out linearization of forces in Eqs. (2) and (3) over drift velocities, we obtain:

$\vec{F}^{(a)}=-e \tilde{\beta}^{(a)} \vec{u}^{(a)} ;$

$\vec{F}^{(a, b)}=-e \tilde{\xi}^{(a, b)}\left(\vec{u}^{(a)}-\vec{u}^{(b)}\right)$.

Here, components of tensors $\tilde{\beta}^{(a)}$ and $\tilde{\xi}^{(a, b)}$ are (see [2, 3] and Eqs. (6) and (7)):

$\beta_{u v}^{(a)}=\lambda_{u v}^{(a)}+\chi_{u v}^{(a)}$;

$\lambda_{u v}^{(a)}=\frac{\hbar}{2(2 \pi)^{5} e n^{(a)} k_{B} T} \int d \omega \times$

$\times \int \frac{q^{2} d^{3} \vec{q}}{\sinh \left(\hbar \omega / k_{B} T\right)} q_{u} q_{v} \operatorname{Im} \Delta \varepsilon_{(a)}^{0}(\omega, \vec{q})\left\langle\varphi_{(I)}^{2}\right\rangle_{\omega, \vec{q}}=$

$=\frac{e n_{I}}{2 \pi^{2} n^{(a)} \varepsilon_{L}^{2}} \int\left[\frac{1}{\omega} \operatorname{Im} \Delta \varepsilon_{(a)}^{0}(\omega, \vec{q})\right]_{\omega=0} \frac{q^{2} q_{u} q_{v}}{\left[q^{2}+q_{0}^{2}(\vec{q})\right]^{2}} d^{3} \vec{q} ;$ 


$$
\begin{aligned}
& \chi_{u v}^{(a)}=\frac{\hbar}{2(2 \pi)^{5} e n^{(a)} k_{B} T} \int d \omega \times \\
& \times \int \frac{q^{2} d^{3} \vec{q}}{\sinh \left(\hbar \omega / k_{B} T\right)} q_{u} q_{v} \operatorname{Im} \Delta \varepsilon_{(a)}^{0}(\omega, \vec{q})\left\langle\varphi_{(p h)}^{2}\right\rangle_{\omega, \vec{q}}= \\
& =\frac{\Xi_{A}^{2} k_{B} T}{32 \pi^{4} n^{(a)} e^{3} \rho s^{2}} \int\left[\frac{1}{\omega} \operatorname{Im} \Delta \varepsilon_{(a)}^{0}(\omega, \vec{q})\right] q_{\omega=0}^{2} q_{u} q_{v} d^{3} \vec{q} ; \\
& \xi_{u v}^{(a, b)}=\frac{\hbar^{2}\left(1-\delta_{a b}\right)}{2(2 \pi)^{4} e \varepsilon_{L}^{2} n^{(a)} k_{B} T} \int \frac{d \omega}{\sinh ^{2}\left(\hbar \omega / 2 k_{B} T\right)} \times \\
& \times \int \frac{q^{4} q_{u} q_{v} d^{3} \vec{q}}{\left[q^{2}+q_{0}^{2}(q)\right]^{2}} \operatorname{Im} \Delta \varepsilon_{(a)}^{0}(\omega, \vec{q}) \operatorname{Im} \Delta \varepsilon_{(b)}^{0}(\omega, \vec{q})
\end{aligned}
$$

In Eqs. (15)-(17), the indices $u, v=x, y, z$ and the imaginary part of dielectric function

$\operatorname{Im} \Delta \varepsilon_{(a)}^{0}(\omega, \vec{q})=-\frac{e^{2}}{\pi q^{2}} \times$

$$
\times \int d^{3} \vec{k}\left[f_{0}\left(\varepsilon_{\vec{k}}^{(a)}\right)-f_{0}\left(\varepsilon_{\vec{k}-\vec{q}}^{(a)}\right)\right] \delta\left(\varepsilon_{\vec{k}-\vec{q}}^{(a)}-\varepsilon_{\vec{k}}^{(a)}+\hbar \omega\right) .
$$
(see [1])

For quasi-elastic collisions, we have the form $\operatorname{Im} \Delta \varepsilon_{(a)}^{(0)}(\omega \rightarrow 0, \vec{q})=\omega \Lambda^{(a)}(\vec{q})$,

where

$$
\begin{aligned}
& \Lambda^{(a)}(\vec{q})=\frac{2 e^{2} m_{\perp} m_{\|}^{1 / 2}}{q^{2} \hbar^{3}}\left(\frac{q_{x}^{2}}{m_{x x}^{(a)}}+\frac{q_{y}^{2}}{m_{y y}^{(a)}}+\frac{q_{z}^{2}}{m_{z z}^{(a)}}\right)^{-1 / 2} \times \\
& \times\left[1+\exp \left\{\frac{\hbar^{2}}{8 k_{B} T}\left(\frac{q_{x}^{2}}{m_{x x}^{(a)}}+\frac{q_{y}^{2}}{m_{y y}^{(a)}}+\frac{q_{z}^{2}}{m_{z z}^{(a)}}\right)-\eta_{a}\right\}\right]^{-1} .
\end{aligned}
$$

As a result, we have:

$$
\begin{aligned}
& \lambda_{u v}^{(a)}=\frac{e n_{I}}{2 \pi^{2} n^{(a)} \varepsilon_{L}^{2}} \int \Lambda^{(a)}(\vec{q}) \frac{q^{2} q_{u} q_{v}}{\left[q^{2}+q_{0}^{2}(\vec{q})\right]^{2}} d^{3} \vec{q}, \\
& \chi_{u v}^{(a)}=\frac{\Xi_{A}^{2} k_{B} T}{32 n^{(a)} \pi^{2} e^{3} \rho s^{2}} \int \Lambda^{(a)}(\vec{q}) q^{2} q_{u} q_{v} d^{3} \vec{q}, \\
& \xi_{u v}^{(a, b)}=\frac{\gamma\left(k_{B} T\right)^{2}}{4 \hbar \pi^{4} e \varepsilon_{L}^{2} n^{(a)}} \int \frac{q^{4} q_{u} q_{v}}{\left[q^{2}+q_{0}^{2}(q)\right]^{2}} \Lambda^{(a)}(\vec{q}) \Lambda^{(b)}(\vec{q}) d^{3} \vec{q},
\end{aligned}
$$

where

$$
\gamma=\int_{-\infty}^{\infty} \frac{w^{2} d w}{\sinh ^{2} w} \approx 3.29
$$

Note that

$$
n^{(a)} \xi_{\alpha \beta}^{(a, b)}=n^{(b)} \xi_{\alpha \beta}^{(b, a)} .
$$

$$
\text { In what follows, we assume } n_{I}=n=\sum_{a=1}^{6} n^{(a)} \text {. }
$$

From Eqs. (1) and (13), one obtains the system of equations for partial drift velocities:

$$
\vec{E}-\tilde{\beta}^{(a)} \vec{u}^{(a)}-\sum_{b=1}^{6} \tilde{\xi}^{(a, b)}\left(\vec{u}^{(a)}-\vec{u}^{(b)}\right)=0
$$

$(a=1,2, \ldots, 6)$.

In this system of equations, kinetic coefficients $\beta^{(a)}$ and $\xi^{(a, b)}$ have a matrix form with matrices $\tilde{\xi}^{(a, b)}$ responding for inter-valley drag. The value $\left(\tilde{\beta}^{(a)}\right)^{-1}$ is the mobility tensor for $a$-carriers, if one neglects intervalley $\operatorname{drag}\left(\tilde{\xi}^{(a, b)} \rightarrow 0\right)$.

\section{Populations of valleys in deformed crystal}

Let a silicon crystal is mechanically compressed along the axis [001] (see Fig. 1), then the components of stress tensor are

$X_{\alpha \beta}=-\delta_{\alpha \beta} \delta_{\alpha z} X$ (here $X>0$ ).

For this situation, the dispersion law for different valleys can be written in the following form (see Ref. [4]):

$\varepsilon_{\vec{k}}^{(a)}(X)=\varepsilon_{\vec{k}}^{(a)}(X=0)+\Delta \varepsilon^{(a)}(X) \equiv \varepsilon_{\vec{k}}^{(a)}+\Delta \varepsilon^{(a)}(X)$ $(a=1,2, \ldots, 6)$,

where

$$
\begin{aligned}
& \Delta \varepsilon^{(1)}(X)=\Delta \varepsilon^{(4)}(X)=-\frac{2}{3} \Xi_{u}\left(s_{11}-s_{12}\right) X, \\
& \Delta \varepsilon^{(2)}(X)=\Delta \varepsilon^{(5)}(X)=\Delta \varepsilon^{(3)}(X)= \\
& =\Delta \varepsilon^{(6)}(X)=\frac{1}{3} \Xi_{u}\left(s_{11}-s_{12}\right) X,
\end{aligned}
$$

$\Xi_{u}$ is the shear deformation potential, $s_{11}$ and $s_{12}$ are the elastic constants.

The density of carriers in the $a$-valley is

$$
\begin{aligned}
& n^{(a)}(X)=\int \frac{d^{3} \vec{k}}{4 \pi^{3}} f_{0}^{(a)}\left(\varepsilon_{\vec{k}}^{(a)}(X)\right)= \\
& =\int \frac{d^{3} \vec{k} / 4 \pi^{3}}{1+\exp \left[\varepsilon_{\vec{k}}^{(a)}(X) / k_{B} T-\eta\right]}= \\
& =\int \frac{d^{3} \vec{k} / 4 \pi^{3}}{1+\exp \left[\left(\varepsilon_{\vec{k}}+\Delta \varepsilon_{\vec{k}}^{(a)}(X)\right) / k_{B} T-\eta\right]} .
\end{aligned}
$$

For nondegenerate carriers

$n^{(a)}(X)=(n / 6)\left[1-\Delta \varepsilon^{(a)}(X) / k_{B} T\right]$.

As it follows from conservation of the total number of carriers (see Eq. (8)),

$$
\begin{aligned}
& \sum_{a=1}^{6} n^{(a)}(X)=\sum_{a=1}^{6} n^{(a)}(0)=n= \\
& =\frac{3\left(2 \pi k_{B} T\right)^{3 / 2} m_{\|}^{3 / 2}}{2 \pi^{3} \hbar^{3} L} F_{1 / 2}(\eta) .
\end{aligned}
$$

\section{Kinetic coefficients for deformed silicon crystal}

Consider the case when the electric field is applied along some fourfold $l$-axis, that is 


$$
\vec{E}=E \vec{e}_{l} \quad(l=x, y, z) .
$$

Then, only $l$-components of drift velocities $\vec{u}^{(a)}$ and diagonal components of the tensors $\tilde{\beta}^{(a)}$ and $\tilde{\xi}^{(a, b)}$ are distinct of zero (in the coordinate system related to fourfold axes), and then one can write the system of equations corresponding to the system (24) in the form$$
E_{l}-\beta_{l l}^{(a)}(X) u_{l}^{(a)}-\sum_{b=1}^{6} \xi_{l l}^{(a, b))}(X)\left(u_{l}^{(a)}-u_{l}^{(b)}\right)=0
$$$$
(a=1,2, \ldots, 6) \text {. }
$$

Here, the expressions for $\beta_{l l}^{(a)}(X)$ and $\xi_{l l}^{(a, b)}(X)$ have the forms (14), (18)-(21) where the dimensionless Fermi energy $\eta_{a}$ in the formulae (18) is shifted in the following manner:

$$
\eta_{a} \rightarrow \eta-\frac{\Delta \varepsilon^{(a)}(X)}{k_{B} T} .
$$

Below we assume the deformation to be small and linearize all the expressions over the stress $X$.

In this paper, we consider only nondegenerate gas (see Fig. 2; there the solid line corresponds to the relation $\left.\varepsilon_{F}(n, T)=0\right)$. Then, it follows from symmetry of the considered system for carriers:

$n^{(a)}(X)=(n / 6) C_{a}(X) ; \quad C_{4}(X)=C_{1}(X) ;$

$C_{3}(X)=C_{5}(X)=C_{6}(X)=C_{2}(X)$,

where (see Eqs. (29), (30))

$$
\begin{aligned}
& C_{1}(X)=1+\frac{2}{3 k_{B} T} \Xi_{u}\left(s_{11}-s_{12}\right) X ; \\
& C_{2}(X)=1-\frac{1}{3 k_{B} T} \Xi_{u}\left(s_{11}-s_{12}\right) X .
\end{aligned}
$$

Note that $C_{1}(X)+2 C_{2}(X)=3$.

Also it follows:

$\beta_{l l}^{(a)}(X)=\beta_{l l}^{(a)}(X=0) ;$

$\xi_{l l}^{(a, b)}(X)=\xi_{l l}^{(a, b)}(X=0) C_{b}(X)$.

Consider now the case when the electric field is applied along $z$-axis, that is

$\vec{E}=E \vec{e}_{z}$.

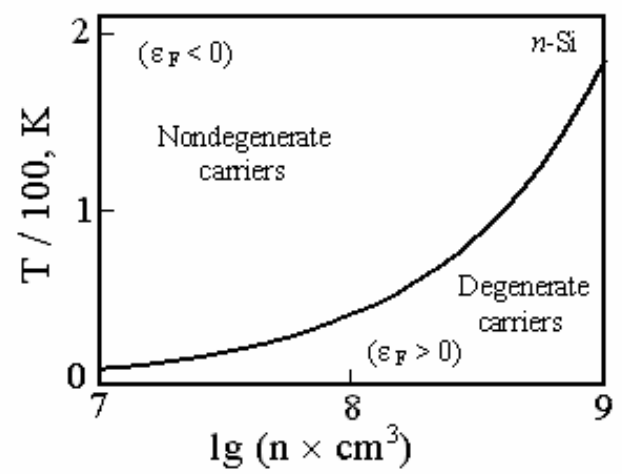

Fig. 2. Areas of degenerate and nondegenerate electron gas.
Then, only $z$-components of drift velocities $\vec{u}^{(a)}$ and $z z$-components of the tensors $\tilde{\beta}^{(a)}$ and $\tilde{\xi}^{(a, b)}$ are distinct of zero, and one can write Eqs. (32) in the form $E_{z}-\beta_{a} u_{z}^{(a)}-\sum_{b=1}^{6} \xi^{(a, b)} C^{(b)}(X)\left(u_{z}^{(a)}-u_{z}^{(b)}\right)=0$ $(a=1,2, \ldots, 6)$.

Here, $\beta_{a}=\beta_{z z}^{(a)}(X=0) ; \xi^{(a, b)}=\xi_{z z}^{(a, b)}(X=0)$.

It is evident that

$$
u_{z}^{(1)}=u_{z}^{(4)} ; \quad u_{z}^{(2)}=u_{z}^{(5)}=u_{z}^{(3)}=u_{z}^{(6)}
$$

and so on. Solving the system (38) and using the relations (39), we obtain $\left(\xi^{(1,2)} \rightarrow \xi\right)$

$u_{z}^{(1,2)}(X ; \xi)=E_{z} \frac{\beta_{2,1}+6 \xi}{\beta_{1} \beta_{2}+2 \xi\left[\beta_{1} C_{1}(X)+2 \beta_{2} C_{2}(X)\right]}$.

One can write the resulting expression for total conductivity of deformed crystal in the following form:

$\sigma_{z z}(X ; \xi)=\frac{j_{z}(X ; \xi)}{E_{z}}=\frac{1}{E_{z}} \sum_{a=1}^{6} j_{z}^{(a)}(X ; \xi)=$

$=\frac{e}{E_{z}} \sum_{a=1}^{6} n^{(a)}(X) u_{z}^{(a)}(X ; \xi)=e n \mu_{z z}(X ; \xi)=$

$=\frac{e n}{3} \frac{\beta_{2} C_{1}(X)+2 \beta_{1} C_{2}(X)+18 \xi}{\beta_{1} \beta_{2}+2 \xi\left[\beta_{1} C_{1}(X)+2 \beta_{2} C_{2}(X)\right]}$.

For undeformed crystal,

$\sigma_{z z}(0 ; \xi)=e n \mu(\xi)=\frac{e n}{3} \frac{\beta_{2}+2 \beta_{1}+18 \xi}{\beta_{1} \beta_{2}+2 \xi\left(\beta_{1}+2 \beta_{2}\right)}$.

Let us define the piezoresistance coefficient $\pi_{k k}(\xi)$ by using the expression (see Refs. $[5,6]$ )

$\pi_{k k}(\xi)=-\frac{1}{\sigma_{k k}(0 ; \xi)}\left[\frac{d \sigma_{k k}(X ; \xi)}{d X}\right]_{X=0}$.

If we direct the electrical field $\vec{E}$ along $x$ - and $y$ axes, for the case (25) we will obtain the relation

$\pi_{z z}(\xi)=-2 \pi_{x x}(\xi)=-2 \pi_{y y}(\xi)$.

To investigate the dependence of conductivity and piezoresistance coefficient on the parameter of intervalley drag, we use the following formulae obtained from the Eqs. (35), (42), (43):

$$
\begin{aligned}
& \frac{\sigma_{z z}(0 ; \xi)}{\sigma_{z z}(0 ; 0)}=\frac{\mu(\xi)}{\mu(0)}=\frac{\beta_{1} \beta_{2}\left(\beta_{2}+2 \beta_{1}+18 \xi\right)}{\left(\beta_{2}+2 \beta_{1}\right)\left[\beta_{1} \beta_{2}+2 \xi\left(\beta_{1}+2 \beta_{2}\right)\right]} ; \\
& \sigma_{z z}(0,0)=\frac{e n}{3} \frac{\beta_{2}+2 \beta_{1}}{\beta_{1} \beta_{2}} \\
& \frac{\pi_{z z}(\xi)}{\pi_{z z}(0)}=\frac{\left(\beta_{2}+2 \beta_{1}\right)\left[\beta_{1} \beta_{2}+6 \xi\left(\beta_{1}+\beta_{2}\right)+36 \xi^{2}\right]}{\left(\beta_{2}+2 \beta_{1}+18 \xi\right)\left[\beta_{1} \beta_{2}+2 \xi\left(\beta_{1}+2 \beta_{2}\right)\right]} \\
& \pi_{z z}(0)=\frac{\Xi_{u}\left(s_{11}-s_{12}\right)}{3 k_{B} T} \frac{2\left(\beta_{2}-\beta_{1}\right)}{\beta_{2}+2 \beta_{1}}
\end{aligned}
$$



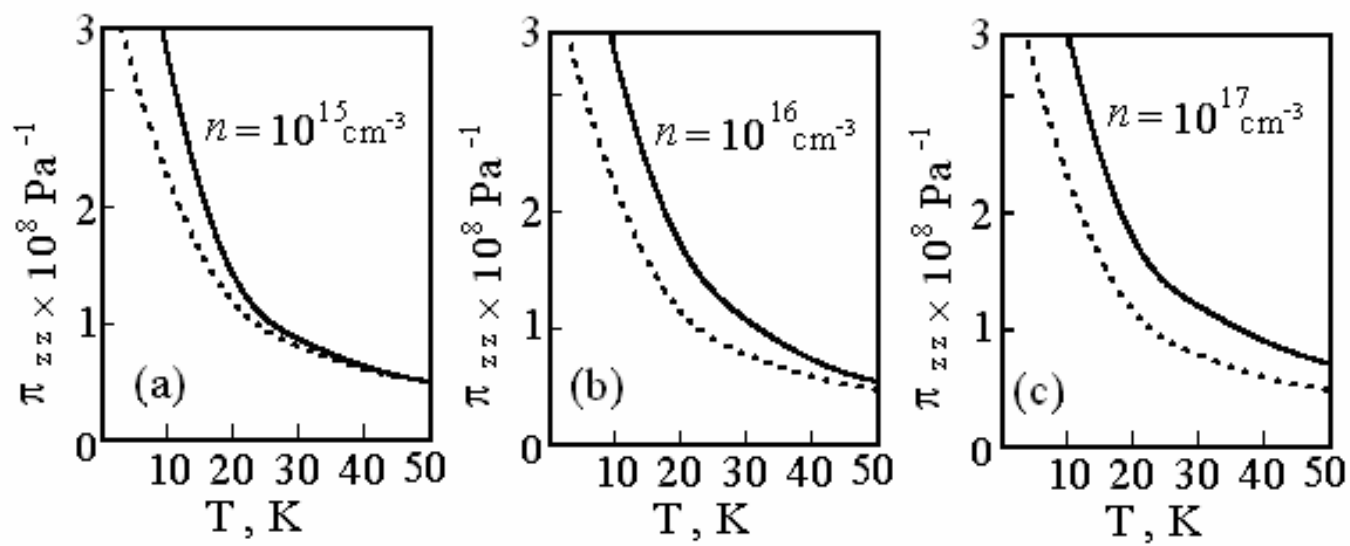

Fig. 3. Dependence of relative piezocoefficient $\pi_{\mathrm{zz}}$ on temperature.

\section{Results of numerical calculations}

These results are shown in Figs 3 to 5. Here, Figs 3(a, $\mathrm{b}, \mathrm{c})$ show the absolute value of piezoresistance coefficients. In calculations, we use the following data (see Refs. [5, 6]): $\quad m_{0}=9.1066 \cdot 10^{-28} \mathrm{~g}$, $m_{\|}=8.342 \cdot 10^{-28} \mathrm{~g} ; \quad M=6 ; \quad L=4.8 ; \quad \varepsilon_{L}=12 ;$

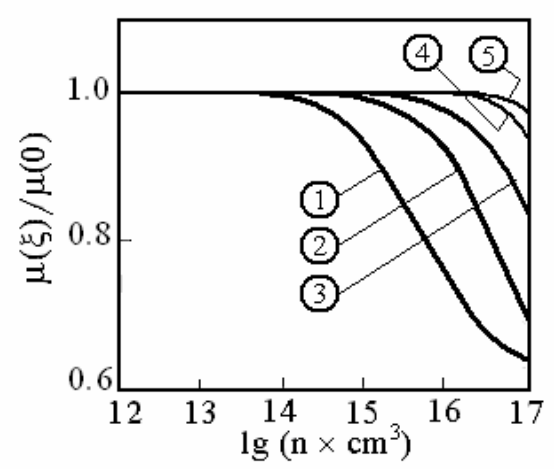

$$
\begin{aligned}
& \left(m_{\|} / m_{0}\right)=0.916 ; \quad s_{11}-s_{12}=9.82 \cdot 10^{-12} \mathrm{~Pa}^{-1}, \\
& \Xi_{u}=8.6 \mathrm{eV} ; \quad \varepsilon_{L}=12, \quad\left(m_{\|} / m_{0}\right)=0.92, \\
& \rho s^{2}=1.66 \cdot 10^{11} \mathrm{~Pa}, \quad \Xi_{A}=\Xi_{d}+(1 / 2) \Xi_{u}=-4.2 \mathrm{eV} \text {. In }
\end{aligned}
$$

Figs (a), (b), (c), solid lines represent the piezoresistance coefficient for crystal where band carriers are involved in drag. The dashed lines correspond to the calculations when inter-valley drag is ignored.

Figs 4 and 5 represent relative values. One can see that inter-valley drag gains the piezocoefficient and diminishes the mobility. Within the region of nondegenerate carriers, the drag effect becomes more pronounced when the carrier concentration grows and temperature falls.

\section{References}

1. I.I. Boiko, Electron-electron drag in crystals with many-valley band // Semiconductor Physics, Quantum Electronics \& Optoelectronics 12 (3), p. 212-217 (2009).

Fig. 4. Dependence of relative mobility on carrier concentration. $1-T=20 \mathrm{~K} ; 2-40 ; 3-70 ; 4-120$; $5-150$.

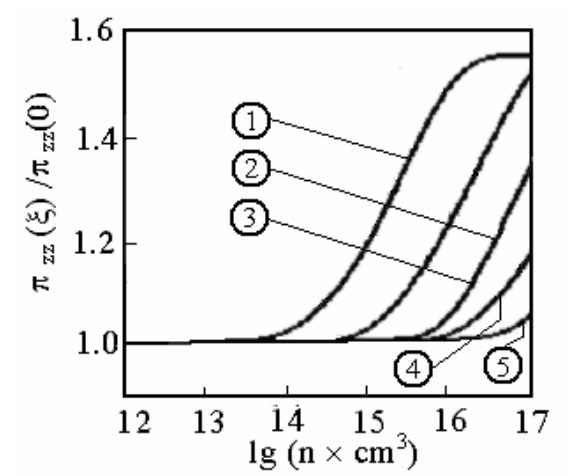

Fig. 5. Dependence of relative piezocoefficient on carrier concentration. $1-T=20 \mathrm{~K} ; 2-40 ; 3-70 ; 4-100$; $5-150$.

2. I.I. Boiko, Transport of Carriers in Semiconductors. Published in V. Lashkaryov Institute of Semiconductor Physics, NAS of Ukraine, Kyiv, 2009 (in Russian).

3. I.I. Boiko and S.I. Kozlovskiy, Investigation of conductivity and piezoresistance of $n$-type silicon on basis of quantum kinetic equation and model distribution function // Sensors and Actuators, A 147, p. 17 (2008).

4. S.I. Kozlovskiy and I.I. Boiko, First-order piezoresistance in silicon crystals // Sensors and Actuators, A 118, p. 33 (2005).

5. P.Y. Yu and M. Cardona, Fundamentals of Semiconductors. Physics and Material Properties. Springer, 2002.

6. C. Herring, E. Vogt, Transport and deformationpotential theory for many-valley semiconductors with anisotropic scattering // Phys. Rev. 101, p. 944 (1956). 